\title{
EchoGéo
}

6 | 2008

La Guyane

\section{Les Africains en Guyane}

Aspects multiformes d'une immigration invisible

\section{André Calmont}

\section{(2) OpenEdition}

Journals

Édition électronique

URL : https://journals.openedition.org/echogeo/6333

DOI : $10.4000 /$ echogeo.6333

ISSN : 1963-1197

Éditeur

Pôle de recherche pour l'organisation et la diffusion de l'information géographique (CNRS UMR 8586)

Référence électronique

André Calmont, « Les Africains en Guyane », EchoGéo [En ligne], 6 | 2008, mis en ligne le 09 juillet 2008, consulté le 31 juillet 2021. URL : http://journals.openedition.org/echogeo/6333 ; DOI : https://doi.org/ $10.4000 /$ echogeo. 6333

Ce document a été généré automatiquement le 31 juillet 2021.

EchoGéo est mis à disposition selon les termes de la licence Creative Commons Attribution - Pas d'Utilisation Commerciale - Pas de Modification 4.0 International (CC BY-NC-ND) 


\title{
Les Africains en Guyane
}

\author{
Aspects multiformes d'une immigration invisible
}

\section{André Calmont}

1 Le caractère polyethnique de la Guyane, héritée d'une longue tradition migratoire, est devenu désormais le trait dominant de la société guyanaise. Les importants flux des années 1970 et 1980 ont sensiblement modifié la mosaïque ethnique, si bien qu'en 1999, les immigrants (Haïtiens, Brésiliens, Français métropolitains, Surinamais, Antillais, Hmongs, pour ne citer que les groupes les plus importants) ont représenté $52 \%$ de la population (Granger, 2006 ; Calmont, 2007). Pourtant, depuis la fin de la décennie 1980, la Guyane a reçu de nouvelles migrations en provenance de la Caraïbe (Dominicains), de l'Amérique du sud (Péruviens) mais aussi de l'Afrique. Encore peu nombreux, les originaires d'Afrique constituent en Guyane une migration originale, très différente des flux régionaux traditionnels : à côté de l'arrivée de sans papiers peu ou pas qualifiés, existe un flux majoritaire de personnes diplômées, arrivées légalement à partir de la France et culturellement proches de la société d'accueil. À cela s'ajoutent une proximité phénotypique et une faible mobilisation des ressources identitaires pour en faire une immigration invisible qui s'insère sans problème majeur dans la société d'accueil.

2 L'étude sur les originaires d'Afrique en Guyane a retenu la zone subsaharienne, excluant les Maghrébins qui n'ont pas la même continuité historique que les autres. Sur le plan méthodologique, nous nous sommes appuyés sur les listes des associations africaines (représentant, à priori, les groupes les plus nombreux) que nous avons complétées par une recherche d'informations sur les Africains en dehors du système associatif. À la suite de cela, nous avons pu établir l'existence de 380 personnes nées en Afrique et résidant en Guyane (enfants non compris). Il est cependant difficile d'estimer l'importance des individus non répertoriés. À partir des listes des associations, nous avons extrait un listing de 78 personnes pour lesquelles nous pouvions disposer d'un certain nombre d'informations et nous avons réalisé des entretiens plus approfondis avec 22 d'entre elles, en juin 2007. Bien entendu, les données présentées ici ne constituent pas une vision absolument représentative des communautés d'origine africaine dans la mesure où l'échantillon reste aléatoire mais les observations faites à partir d'elles peuvent être retenues comme des indications et des tendances. 


\section{Une double dimension migratoire}

\section{Dans le passé, une immigration contractuelle}

Au milieu du XIX ${ }^{\mathrm{e}}$ siècle, dans la période post-abolitionniste, la Guyane avait déjà connu l'arrivée d'Africains, dans le cadre d'une immigration contractuelle. En effet, après l'abolition de l'esclavage en 1848 , les nouveaux libres affluèrent à Cayenne ou s'installèrent loin des zones habitées pour pratiquer l'abattis ou encore, pour ceux qui possédaient des économies, achetèrent à leur ancien maître un lopin de terre. Malgré toutes les mesures répressives visant à maintenir de force les affranchis sur les habitations, le travail ne reprit pas et il fallut envisager l'immigration pour faire face au manque de main-d'œuvre sur les exploitations. À la suite de l'échec d'une introduction de Madériens (248 sont arrivés entre 1849 et 1851), la Guyane se tourna vers les travailleurs africains : de 1854 à 1859, arrivèrent 1969 Africains, en sept convois recrutés dans la population libre ou formés de captifs rachetés et rendus à la liberté, pour des contrats de travail de cinq ans. La fin de l'immigration africaine intervint dès 1860 sous la pression des autorités britanniques qui y voyaient une forme de traite déguisée $^{1}$. Les rapatriements en fin de contrat et surtout une mortalité importante réduisirent considérablement la communauté si bien qu'en 1866, il ne restait plus que 947 inscrits sur le registre des immigrants mais, en réalité, le nombre d'Africains en Guyane était plus important, les autres étant rattachés à la population créole à laquelle ils s'étaient parfaitement intégrés (Girondin, 1986).

4 Soixante dix ans plus tard, on assista à l'arrivée d'autres Africains en Guyane, mais dans le cadre d'une installation provisoire. En effet, un bataillon de 150 tirailleurs " sénégalais » (en réalité, ils venaient de différents pays d'Afrique) avait été installé en Guyane pour compléter les forces de l'ordre, depuis les émeutes de 1928 provoquées par la mort suspecte de Jean Galmot, homme politique et homme d'affaires guyanais ${ }^{2}$. La relève du bataillon avait été rendue impossible depuis 1940 à cause de la guerre mais elle tardait à s'effectuer depuis la fin des hostilités, et le mécontentement des militaires africains était d'autant plus grand qu'ils avaient le sentiment de ne pas être aimés par la population guyanaise qui les percevait en effet comme des êtres frustres et peu civilisés. Aussi, le 24 février 1946, un incident à la sortie d'un bal de carnaval servit d'exutoire aux tirailleurs et déboucha sur deux jours d'émeutes qui se soldèrent par 8 victimes et une cinquantaine de blessés. Le bataillon fut alors très rapidement évacué du pays ${ }^{3}$.

\section{Des migrations légales à partir de la France}

5 À l'époque contemporaine, depuis la départementalisation, l'arrivée d'Africains à nouveau en Guyane date des années $1980^{4}$ mais le mouvement s'est accentué depuis la décennie 1990 (six personnes interrogées sur dix sont arrivées après 2000). Le phénomène revêt une double dimension migratoire, au départ de la France et au départ de l'Afrique.

6 L'essentiel du flux est formé par une migration légale en provenance de la France et constitué par des personnes qualifiées, médecins et enseignants en particulier. Le choix de la Guyane s'explique par trois facteurs: d'abord les possibilités d'emploi et les 
perspectives de carrière plus rapides qu'ailleurs en raison du manque important de personnel qualifié dans ce pays, ensuite l'aspect financier puisque les fonctionnaires y perçoivent une rémunération très supérieure à celle qu'ils auraient touchée en France métropolitaine, non seulement un supplément de $40 \%$ sur les salaires mais également le versement d'une prime substantielle pour la nomination en Guyane (cette prime qui existait dans tous les départements d'outre-mer a été supprimée récemment aux Antilles), et enfin l'éloignement de frustrations et d'absence de valorisation que les Africains vivent dans l'Hexagone, la migration pouvant être interprétée comme celle de cadres à la recherche d'une reconnaissance, plus facile à obtenir en Guyane. Pour certains, la Guyane n'était qu'une possibilité parmi d'autres choix de mutation dans l'outre-mer français (l'une des personnes interrogées pensait, avant de venir, que la Guyane était une île de la Caraïbe comme les Antilles).C'est donc après une période d'activité professionnelle en France métropolitaine que les Africains décident de s'installer en Guyane.

7 Concernant le choix de la Guyane plutôt que le retour au pays, les situations sont également fort complexes et les motivations se superposent : le pays visé par le migrant apparaît attractif et, en parallèle, le pays africain d'origine semble répulsif, en raison de la situation économique (pas de possibilité d'emploi, pas d'emploi dans de bonnes conditions, perte des avantages en matière de retraite en cas de retour en Afrique), du contexte politique (situation de crise, absence de liberté, crainte pour ceux qui avaient milité en France contre le pouvoir en place), de la situation culturelle (éloignement de la pression de la famille et de la tradition), de la difficulté pour le conjoint non africain de s'insérer socialement ou économiquement dans le pays de l'époux.

8 Les résultats de l'enquête montrent qu'une grande partie des originaires de l'Afrique subsaharienne venant de France possède la nationalité française. Si quelques uns ont uniquement la nationalité française, la plupart possèdent la double nationalité, voire la triple nationalité, lorsqu'ils ont vécu ou grandi dans un pays africain voisin de celui de leur naissance (Togo/Bénin, Sénégal/Cap Vert ou Sénégal/Mali par exemple). Parmi ceux qui ont la nationalité française, il y a les personnes qui la possèdent depuis l'indépendance de leur pays tandis que les autres l'ont obtenue pour longue résidence en France ou par mariage.

\section{Un flux de sans papiers au départ de l'Afrique}

9 Il existe également une migration de sans papiers, certes marginale par rapport à la migration légale, qui vient d'Afrique et dont la destination finale reste la France métropolitaine. La filière passe par le Brésil, au départ de Saõ Tomé (pas de visa exigé au départ de ce pays pour l'entrée au Brésil) ou de Dakar ou même de l'Angola (comme ce fut le cas pour un groupe de 14 Congolais de la RDC arrivés en Guyane en 2000), par avion ou parfois par bateau. Aux différentes étapes du voyage (Fortaleza, Belém, Macapá, Oïapoque), les clandestins sont hébergés par des Africains résidant dans ces villes, avant d'arriver à Cayenne où ils sont pris en charge par des compatriotes ou bien, dans les situations extrêmes, par des associations caritatives ou africaines.

On compte ainsi plusieurs dizaines de sans papiers, les chiffres avancés par nos informateurs allant d'une trentaine à plus d'une centaine, mais les demandes de régularisation sont souvent satisfaites. Les clandestins sont surtout des Sénégalais mais 
aussi des Cap Verdiens, des Ivoiriens, des ressortissants de Guinée Bissau et même des Ghanéens, ces derniers utilisant plutôt la filière par le Surinam. Le développement de la migration illicite africaine vers la Guyane est le résultat de l'aggravation de la situation économique (comme au Sénégal) ou politique (Côte d'Ivoire) des pays africains. À cela, s'ajoute le fait que, depuis les années 2000 , il devient de plus en plus difficile pour les sans papiers de se rendre directement en France.

11 Arrivés en Guyane, nombreux sont les clandestins qui font une demande d'asile auprès de l'OFPRA (Office français de protection des réfugiés et apatrides) dont ils savent que l'attente de la réponse, souvent plus d'un an, leur permettra de rester légalement dans le pays pendant un certain temps 5 . En réalité, la plupart d'entre eux sont des réfugiés économiques, y compris les Ivoiriens, car les vrais réfugiés politiques se rendent directement dans l'Hexagone. Lorsqu'ils finissent par régulariser leur situation, ils restent en Guyane et c'est souvent le cas des Ivoiriens, à la fois en raison de la situation politique de leur pays mais aussi du fait que la plupart d'entre eux possèdent une formation, un métier ou même des diplômes. Dans le cas contraire, les sans papiers repartent dans leur pays d'origine (ils y sont d'ailleurs incités par les associations africaines de Guyane) : ils travaillent dur, notamment dans l'orpaillage, et se sacrifient pendant un ou deux ans pour faire des économies afin de repartir, d'autant plus que certains ont laissé une femme "au pays ». Les autres stratégies pour régulariser sa situation et rester légalement en Guyane consistent à obtenir un certificat d'embauche de son employeur, se marier avec une personne de nationalité française ou même encore à se procurer illégalement une carte de séjour.

Mais la situation de ces migrants reste toujours précaire, comme le montre l'affaire du squat en décembre 2006 : un vieil immeuble du centre-ville de Cayenne avait été occupé par plusieurs dizaines de sans-papiers d'origine africaine, avec l'accord plus ou tacite de la propriétaire, mais le nouveau propriétaire a cherché à les expulser pour réhabiliter le bâtiment et la quarantaine de clandestins restants se sont retrouvés ainsi à la rue. L'Évêché et une association africaine sont alors intervenus pour trouver une solution d'hébergement pour les clandestins, ainsi q'une aide financière et administrative (régularisation).

Cependant, la migration clandestine tend, semble-t-il, à diminuer. Il faut dire que depuis l'affaire du marché de Cayenne en septembre $2006^{6}$, les associations africaines incitent les migrants à ne pas favoriser la venue d'autres migrants, qui risquerait de créer une situation difficile. On note par ailleurs que ceux qui arrivent depuis fin 2006 sont très jeunes, moins de 18 ans, et ils peuvent donc s'insérer dans les circuits scolaires ou de formation et sont ainsi plus difficiles à expulser. De toutes façons, le reconduites à la frontière d'Africains sont très rares en raison du coût de l'opération et de la discrétion de ces migrants (il n'y a eu que deux reconduites à la frontière ces trois dernières années).

\section{Une migration limitée mais qualifiée}

\section{Aspects sociodémographiques de la communauté africaine}

14 Les Africains installés en Guyane sont originaires d'une vingtaine de pays dont une dizaine à présence significative. Les Sénégalais apparaissent nettement les plus nombreux et, avec près d'une centaine de personnes, ils sont deux fois plus nombreux 
que le groupe suivant et représentent plus du quart des originaires du continent noir (figure 1). Parmi les autres pays qui rassemblent plusieurs dizaines de ressortissants, se détache la Côte d'Ivoire d'où les gens migraient peu auparavant, compte tenu du dynamisme économique et de la stabilité politique du pays jusqu'au début des années 1990. Au niveau de la répartition géographique, la région de Cayenne rassemble plus de la moitié de la communauté, les deux autres villes de Saint-Laurent et de Kourou se partageant équitablement le reste, en dehors d'une ou deux dizaines de personnes éparpillées sur le reste du territoire ${ }^{7}$.

Figure 1

Les communautés d'origine africaine en Guyane

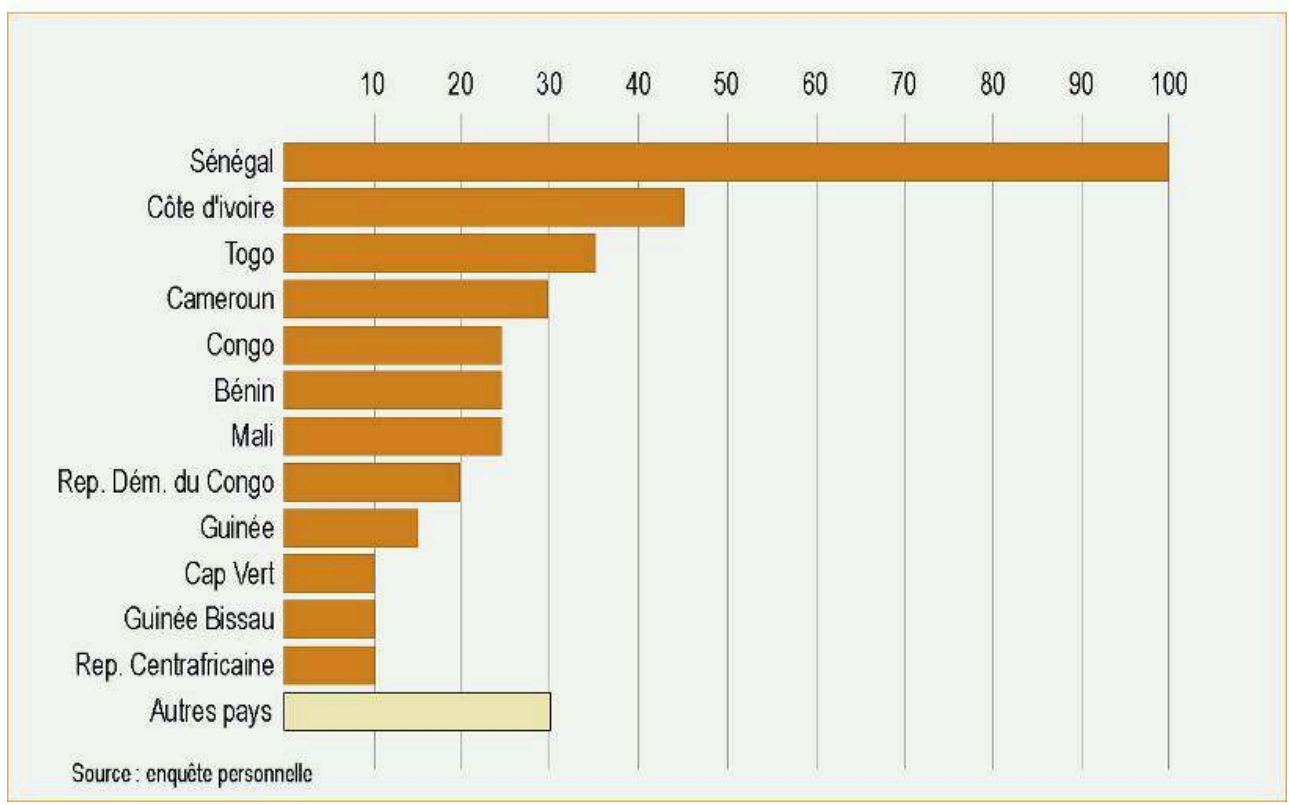

Les migrants, qui appartiennent à deux catégories d'âge selon le type de migration (16 à 35 ans pour les sans papiers et, pour ceux arrivés dans un cadre légal, plus de 30 ans et généralement entre 40 et 50 ans) sont majoritairement des hommes (deux tiers des effectifs), les femmes étant surtout à Kourou, mariées à des Français et souvent sans profession. Cependant, nombre d'Africaines ont également un niveau universitaire et sont médecins, enseignantes ou avocates ${ }^{8}$.

Les couples endogames sont finalement assez peu nombreux, un tiers des 32 couples répertoriés, mais ce pourcentage est cependant le double de celui constaté aux Antilles françaises ${ }^{9}$, tandis que les couples franco-africains ${ }^{10}$ sont presque aussi nombreux et que ceux qui comptent un conjoint guyanais, ces derniers représentant un quart de l'effectif (contre $60 \%$ dans les départements antillais) ${ }^{11}$. La faible endogamie s'explique dans la mesure où l'immigration de célibataires est exclusivement masculine, la plupart des femmes africaines étant mariées à des non Africains, des Français ou des Antillais qui ont obtenu une mutation en Guyane comme les fonctionnaires ou qui ont été recrutés depuis la France comme les médecins. L'ancrage familial moins profond en Guyane qu'aux Antilles se traduit par une plus forte mobilité des Africains. Sur place, les Africains célibataires trouvent une compagne créole, guyanaise ou antillaise, beaucoup plus rarement brésilienne, ce qui facilite la régularisation.

Dans la quasi totalité des couples guyano-africains, c'est l'époux qui est africain alors que c'est l'inverse pour les couples franco-africains où la femme est africaine dans sept 
cas sur dix. Cet aspect est important car les femmes et les hommes ne jouissent pas du même prestige au sein de la société guyanaise. L'insertion apparaît, semble-t-il, plus poussée lorsque, dans le couple, c'est l'homme qui est d'origine africaine mais il est vrai que d'autres facteurs peuvent interférer, par exemple l'intégration est facilitée lorsque le niveau de compétence est élevé. Cependant, à l'intérieur de la population féminine, les situations divergent selon le niveau de diplôme.

\section{Une migration de personnes qualifiées}

Les résultats de l'enquête montrent que les migrants ont un niveau de qualification élevé puisque les deux secteurs d'activité qui dominent largement sont l'enseignementformation ( $22 \%$ des personnes répertoriées) et le domaine médical et paramédical $(13 \%)$, totalisant plus du tiers de l'éventail socio-économique. Le profil est ainsi très différent de celui des Africains en Europe mais proche de ceux installés aux États-Unis (Diouf-Kamara, 1991). La communauté semble peu touchée par le chômage puisque la catégorie des "sans profession » ne représente qu'un dixième de l'effectif, d'autant plus que la plupart des personnes sans activité sont des épouses au foyer, qui ne cherchent pas d'emploi. Même les sans papiers, qui ne sont pas venus ici pour les facilités sociales de la Guyane, ont ou cherchent généralement une activité.

Les enseignants sont de loin les plus nombreux car c'est le marché de l'emploi qui prime sur les autres considérations. En effet, même si beaucoup d'Africains préfèrent la France, les carrières s'avèrent en revanche plus faciles et plus rapides en Guyane que dans l'Hexagone et même qu'aux Antilles, compte tenu du déficit important dans cette branche. Ayant fait des études supérieures poussées, ils se retrouvent surtout dans le cycle secondaire (collège, lycée) et aussi supérieur (une demi-douzaine exerce à l'antenne guyanaise de l'Université) mais on trouve également des professeurs des écoles ainsi que des personnels d'administration. Comme pour l'ensemble de la communauté, les enseignants exercent surtout dans les trois villes de Guyane mais aussi dans le reste du pays, y compris « sur le fleuve».

Dans le domaine médical, la très grande majorité est constituée par les médecins, près d'une trentaine, surtout dans les établissements hospitaliers (Denour et Junker, 1995). $\mathrm{Si}$ on prend l'exemple de Saint-Laurent, sur les 17 personnes d'origine africaine travaillant dans le secteur de la santé, on compte 11 médecins (6 à l'hôpital, 4 médecins libéraux et un pédiatre en PMI), les 6 autres personnes travaillant à l'hôpital ou dans d'autres secteurs de la santé (pharmacien, dentiste, radiologue) ${ }^{12}$. Le paradoxe, c'est que l'Afrique doit faire face à une désastreuse situation sanitaire, alors que 20000 professionnels de santé émigrent chaque année de ce continent vers l'Europe ou l'Amérique du Nord. Cette migration qualifiée représente un manque à gagner pour les pays d'origine au niveau du coût de la formation et, sur le plan sanitaire, l'exode des cerveaux a des conséquences catastrophiques (Blanchet et Keith, 2006).

Mis à part les domaines de la santé et de l'éducation, l'éventail socioprofessionnel est devenu beaucoup plus large qu'au début de la migration. On trouve en effet un nombre important d'employés et aussi de cadres dans la fonction publique (État et collectivités locales) ou dans le secteur privé. Si les activités commerciales apparaissent presque aussi nombreuses, il s'agit en réalité, dans la plupart des cas, de quelques commerces ethniques (vente de vêtements et de produits artisanaux ainsi qu'un restaurant proposant de la cuisine africaine) mais surtout de commerçants ambulants qu'on 
trouve aux abords des marchés de Cayenne et de Kourou. On trouve par ailleurs de plus en plus d'Africains dans le secteur de l'orpaillage car c'est une stratégie pour la régularisation que de se présenter comme recherchant un emploi dans une branche où il y a insuffisance de main-d'œuvre locale. S'il y a très peu de techniciens ou de chefs d'entreprise, on trouve en revanche quelques professions libérales mais aussi des artisans et même deux marins pêcheurs, ainsi que certains créneaux spécifiques comme la religion (prêtre, pasteur, imam). Le secteur des "petits boulots " complète le panorama socioprofessionnel. En dehors du marché, ils travaillent également dans les restaurants et les épiceries de la communauté chinoise ou sont laveurs de voitures ${ }^{13}$.

Les marabouts constituent un cas particulier. Une dizaine d'entre eux proposent leurs services de voyance, essentiellement des Guinéens et des Maliens mais aussi des Sénégalais, en concurrence avec d'autres d'origine française, haïtienne ou guyanaise. Les marabouts sont généralement originaires de l'Afrique sahélienne, très tôt pénétrée par l'islam ${ }^{14}$. Les marabouts sont arrivés en France dans le sillage des premiers migrants de l'Afrique de l'Ouest que furent les Soninké et ils s'inscrivent dans le monde de la voyance pratiquée en France. Si quelques uns sont installés dans le pays, les autres marabouts sont plus mobiles: résidant aux Antilles françaises, en provenance de France, ils viennent exercer en Guyane pour des périodes de quelques semaines.

\section{Les Africains de Guyane et l'Afrique}

\section{Les transferts financiers de la migration}

Les rapports que les Africains entretiennent avec leur pays d'origine sont d'ordre affectif (les retours "au pays») et financiers (les remises). Le retour au pays natal s'effectue, pour ceux qui le peuvent, tous des deux ou trois ans, parfois à un rythme plus espacé, en raison du coût du voyage au départ de la Guyane, surtout quand on part en famille. Quelques uns se contentent parfois de se rendre seulement dans l'Hexagone où ils ont de la famille. En tout cas, presque tous envisagent le retour définitif au moment de la retraite et certains y ont déjà réalisé un investissement immobilier dans cette perspective. De toutes façons, pour beaucoup, les enseignants en particulier, le séjour en Guyane est perçu comme temporaire, en moyenne cinq ou six ans où bout desquels ils partent pour retourner en France métropolitaine ou aller dans l'outre-mer français, davantage Mayotte ou la Polynésie que les Antilles (où les postes disponibles sont rares et la prime absente).

Mais les relations que les Africains entretiennent avec le pays natal sont constituées surtout par les remises ou transferts financiers. La plupart des Africains envoient de l'argent à leur famille, en dehors de ceux qui sont issus des classes moyennes et dont les parents sont à l'abri du besoin. Paradoxalement, ce sont les sans papiers qui effectuent les envois les plus réguliers, même s'il s'agit de montants modestes. La migration illicite est en effet souvent une stratégie de la famille qui choisit un membre pour tenter l'aventure, finance le voyage, à charge pour le migrant, une fois installé à l'étranger, de subvenir aux besoins de ceux qui sont restés, les remises étant donc une obligation de retour.

L'argent est essentiellement destiné aux besoins quotidiens, en particulier la santé et la scolarisation, qui sont importants compte tenu de la taille des familles, ce qui laisse peu de place pour les investissements productifs. Il est vrai que l'implication économique 
des Africains est faible lorsque l'argent à investir vient de la diaspora dans la mesure où on n'a pas vraiment à rembourser le capital d'origine familiale. Il est vrai aussi que l'activité économique est difficile dans le contexte social et économique des pays africains. Ce sont les ressortissants du Sénégal qui envoient le plus d'argent et le plus régulièrement car dans un pays de solidarité comme celui-là, "l'impôt social » est considéré comme normal et même moralement obligatoire, l'argent de la diaspora permettant de faire face aux situations les plus difficiles. Ainsi, le Sénégal reçoit globalement plus de 340 millions de dollars de la diaspora (Charbit et Chort, 2006), ce qui représente $7 \%$ du PIB national et $35 \$$ par habitant et par an (tableau 1).

Tableau 1 Transferts des migrants reçus dans des pays africains en 2003

\begin{tabular}{|c|c|c|c|}
\hline pays d'origine des migrants & $\begin{array}{c}\text { transferts des migrants } \\
\text { en millions de } \$\end{array}$ & $\begin{array}{c}\text { transferts des migrants } \\
\text { en \% du PIB }\end{array}$ & $\begin{array}{c}\text { transferts des migrants } \\
\text { en } \$ \text { par habitant }\end{array}$ \\
\hline Sénégal ${ }^{\star}$ & 344,5 & 6,84 & 34,96 \\
\hline Côte d'Ivoire & 141 & 0,96 & 8,48 \\
\hline Mali* $^{*}$ & 137,6 & 4,37 & 10,91 \\
\hline Cap Vert & 92,2 & 11,28 & 199,16 \\
\hline Bénin ${ }^{\star *}$ & 83,6 & 3,53 & 13,09 \\
\hline Togo** & 68,5 & 5,15 & 14,62 \\
\hline Ghana & 65,1 & 0,88 & 3,11 \\
\hline Burkina Faso** & 49,9 & 2,02 & 4,07 \\
\hline Guinée* & 15,2 & 0,47 & 1,82 \\
\hline Congo* & 1,2 & 0,04 & 0,32 \\
\hline
\end{tabular}

$\left.{ }^{*}\right)$ données 2002

(*) données 2001

Des indications chiffrées ont été fournies au cours des entretiens et elles vont de $1000 €$ à $3000 €$ par an et par ménage expatrié. En extrapolant les données, on arrive à un montant d'environ un demi million d'euros par an pour les transferts monétaires de la Guyane vers les pays africains.

\section{La gestion des ressources identitaires}

La mobilisation des ressources identitaires peut s'analyser à travers les pratiques linguistiques, religieuses et vestimentaires. Au quotidien, les Africains de Guyane s'habillent très rarement "à l'africaine", pour sortir ou aller travailler, compte tenu sans doute de leur niveau socioculturel et donc de leur occidentalisation, sauf seulement lors des grandes occasions (baptême par exemple). Il est vrai que, même en France, ce sont les personnes peu intégrées, de niveau socioéconomique faible, les femmes surtout, qui s'habillent au quotidien de manière traditionnelle, la communauté la plus portée à se vêtir ainsi étant les Maliens.

Dans ce contexte là, la langue est-elle un marqueur d'identité ? Quand les Africains rencontrent des compatriotes, ils communiquent dans la langue du pays d'origine mais rapidement, c'est le français qui s'impose dans la conversation, phénomène plus fréquent pour les originaires de l'Afrique centrale (où il n'existe que peu de langues fédératrices) que de l'Afrique sahélienne. Ainsi, en République Démocratique du Congo (ex Zaïre) par exemple, à côté de la langue française qui est prééminente dans la vie publique et officielle, il existe quatre langues nationales et une multiplicité de langues régionales. Les quatre langues nationales sont réparties selon les régions et l'une d'entre elles est enseignée à l'école. Il y a le kikongo à l'ouest (langue partagée avec le Congo Brazza et l'Angola), le kiluba dans le centre du pays, le lingala dans la partie nord 
(langue qui s'est imposée comme celle de l'armée et de la musique congolaise) et le swahili dans tout l'est du pays.

Sur le plan religieux, l'islam constitue une ressource identitaire pour les originaires de l'Afrique sahélienne (Sénégal, Mali, Guinée mais aussi une partie croissante du Bénin, de la Côte d'Ivoire). Ceux-ci se rendent, notamment le vendredi, jour de la grande prière, à la mosquée (plus un lieu de prière qu'une véritable mosquée), dans la banlieue de Cayenne. La communauté musulmane en Guyane compterait 750 fidèles dont un quart originaire de l'Afrique subsaharienne (et une quinzaine de Créoles guyanais), les autres étant non seulement des Maghrébins et des Levantins (Syriens, Libanais) mais aussi et surtout des Surinamais et des Guyaniens dont les pays connaissent une implantation de l'islam très ancienne et très importante (l'imam et les quatre vice imams sont cependant tous sénégalais). À la différence de la France métropolitaine, la présence de l'islam, peu présent ici il est vrai (moins de $0,5 \%$ de la population) ne pose aucun problème dans une Guyane multiculturelle. Il reste que la mosquée devient un lieu de sociabilité pour les Africains musulmans, au même titre que le restaurant africain de Cayenne où les Sénégalais ont pris l'habitude de se rencontrer, certains étant même hébergés dans l'immeuble au moment de leur arrivée en Guyane.

\section{Les Africains et la Guyane}

\section{L'insertion des Africains dans la société guyanaise}

Elle semble globalement bonne puisque les deux tiers des personnes interrogées estiment n'avoir pas (eu) de problèmes d'intégration, même si certains pensent qu'il y a un certain rejet de l'Afrique, peu verbalisé mais réel (nombre d'enseignants admettent qu'ils sont parfois appelés "l'Africain» par leurs élèves, avec une connotation péjorative et, même dans la région du Maroni, des élèves et parents d'élèves disent préférer des enseignants français métropolitains aux Africains. Ces derniers sont cependant perçus différemment selon leur statut («Il est africain mais c'est un médecin ») et selon le niveau socioculturel des Guyanais (ils sont mieux acceptés par les catégories sociales élevées de la population). Comment interpréter cette attitude? Est-ce que le Guyanais n'a pas totalement intégré son africanité, ressentiment davantage que sentiment anti-africain, en partie lié à l'esclavage ("Vous nous avez vendus»), d'autant plus que le pays a reçu, depuis, de très nombreux autres apports démographiques ou bien est-ce qu'il est victime des stéréotypes et de l'image négative de l'Afrique véhiculés par les médias, sans doute renforcés ici par la transmission générationnelle de l'affaire des tirailleurs sénégalais?

Il reste cependant que, d'une manière générale, l'immigration africaine, même illicite, n'est pas stigmatisée par la population guyanaise. Il est vrai qu'ils sont encore peu nombreux, ne sont guère impliqués dans les faits de délinquance et sont perçus comme des individus travailleurs, "souples" et peu vindicatifs ${ }^{15}$. Il est vrai aussi que les étrangers diplômés sont regardés positivement, se différenciant des flux de migrants non qualifiés à la charge des organismes sociaux.

31 Les rapports entre les "Africains de l'Afrique actuelle " et les Bushinenge, seuls descendants non métissés des Africains de la période esclavagiste ${ }^{16}$, sont empreints d'une émotion mutuelle, chez les Bushinenge de rencontrer les Africains descendants de leurs ancêtres, émotion ressentie également chez les Africains de rencontrer leurs 
«frères » africains du Nouveau monde, d'autant plus qu'ils constatent des survivances très fortes de l'Afrique dans la communication sociale, les contes funéraires, les chants ou les danses (Moomou, 2007). Un Béninois, enseignant «sur le fleuve », a emmené en 2006 un groupe de 15 adultes bushinenge dans son pays natal, voyage initiatique historico-culturel très fort (le Bénin est le berceau du vaudou et Ouidah, dans ce pays, a été, avec Gorée, un des principaux ports négriers) qui a largement démythifié l'Afrique auprès de cette catégorie de population qui, comme les autres Guyanais, ne perçoit le continent noir que comme un espace de pauvreté et de famine, très en retard par rapport à la modernité.

\section{Le mouvement associatif africain}

Le mouvement associatif permet non seulement aux groupes de migrants de se retrouver mais aussi de se visibiliser dans la société d'accueil et les Africains n'échappent pas à la règle. Il existe quatre associations d'Africains en Guyane, trois organisations panafricaines et une association « nationale ", celle des Sénégalais. Celleci, appelée « Téranga » et créée en 2005, est avant tout une amicale avec des activités ludiques qui permettent aux originaires du Sénégal de se retrouver dans le cadre de soirées dansantes, de manifestations musicales ou gastronomiques.

Il existe à Cayenne deux associations panafricaines, avec chacune des objectifs différents. L'association Afrique-Guyane a été constituée en 2004 pour créer un espace de rencontre entre les Africains du pays, notamment pour les enfants des communautés africaines. Les activités de l'association sont sportives, sociales et culturelles : projection de films sur les réalités africaines, soirée dansante, randonnée en forêt, sortie à la plage,... L'association compte une cinquantaine de membres dont les trois quarts sont Africains, les autres étant très fréquemment les conjoints des Africains ou bien alors des personnes, surtout des Français métropolitains, qui ont séjourné en Afrique. L'association joue également un rôle important dans l'encadrement des migrants en difficulté : intervention auprès de la Préfecture lorsque les clandestins arrêtés se retrouvent au centre de rétention de Rochambeau dans l'attente d'une reconduite à la frontière, soutien matériel et psychologique des Africains en prison (certains y sont pour trafic de drogue), suivi des dossiers de demande de régularisation, soutien matériel à ceux qui sont dans les situations les plus critiques. Pour certaines de ces actions, Afrique-Guyane travaille en collaboration avec la Cimade ${ }^{17}$ qui a une antenne en Guyane et qui suit le traitement des affaires de migrants clandestins. À plus long terme, l'association souhaite réaliser des opérations pour aider au développement de l'Afrique mais l'unanimité est loin de se faire sur des projets.

L'autre regroupement, le CEDIPA (Cercle d'initiative pour l'Afrique), créée en 2005, est plus engagé : l'article II de ses statuts rappelle que «l'association a pour objectifs [d'abord] de défendre la mémoire des esclaves et l'honneur de leurs descendants, de combattre le racisme et d'assister les victimes de discrimination raciale, [ensuite] de promouvoir et de défendre l'histoire, la culture et l'identité africaines». Sa principale activité, pour le moment, consiste dans l'organisation de colloques internationaux en rapport avec l'Afrique (" esclavage et mémoire » en 2006 puis « L'Afrique dans la traite négrière » en 2007) qui se passent lors de la commémoration de l'abolition de l'esclavage en Guyane (10 juin). Cependant, le CEDIPA a été amené également à prendre en charge des migrants en difficulté matérielle et/ou administrative. 
La troisième association panafricaine, l'ADAM (Association des Africains du Maroni) est centrée sur la ville de Saint-Laurent et a un double objectif : d'abord créer un lien de solidarité entre ses membres car certains Africains, souvent sans leur famille, se sentent isolés, et ensuite participer à la vie saint-laurentaise (don de livres de littérature africaine à la bibliothèque municipale, participation à la fête annuelle de Saint-Laurent où l'association tient un stand et anime une soirée culturelle consacrée à l'Afrique).

Les associations regroupent cependant une petite fraction de la communauté car les Africains installés en Guyane ne recherchent pas systématiquement les compatriotes et certains, qui estiment que la vie associative participe au communautarisme, ne veulent pas exister en tant qu'Africains, invisibilité qui leur permet de s'intégrer d'autant plus rapidement. Il est vrai que la diversité et la nature des professions représentées en Guyane ne sont pas favorables à la dynamique associative, ce type de regroupement étant surtout le fait de ceux qui sont ou peuvent être confrontés à des problèmes d'insertion (absence ou renouvellement de titre de séjour, recherche d'un emploi, problème de discrimination), ce qui n'est pas souvent le cas des originaires d'Afrique en Guyane.

En fin de compte, les Africains, arrivés en Guyane depuis une vingtaine d'années, constituent, en raison des caractéristiques spécifiques de cette migration (faible importance numérique, niveau de qualification élevé, proximité phénotypique et culturelle) un groupe invisible dans la société guyanaise et même la migration illicite n'est pas stigmatisée par la population locale. Cependant, la mobilité de cette communauté reste beaucoup plus forte qu'aux Antilles dans la mesure où l'ancrage familial est moins intense, mais aussi dans la mesure où beaucoup d'Africains viennent en Guyane pour profiter des meilleures perspectives de carrière et de salaire ou comme espace de transit avant une réémigration vers la France métropolitaine. Il ne reste pas moins vrai que, dans un pays peu peuplé et déficitaire en personnels qualifiés dans de nombreux secteurs, une plus grande sédentarisation de cette migration pourrait être un élément, parmi d'autres, d'une dynamique de développement de la Guyane.

\section{BIBLIOGRAPHIE}

Blanchet K. et Keith R., 2006, L'Afrique tente de retenir ses médecins. Le monde diplomatique, décembre, p.13.

Calmont A., 2007, Dynamiques migratoires en Guyane : des politiques migratoires de développement au développement des migrations spontanées. Comprendre la Guyane d'aujourd'hui, Ibis Rouge Editions, Matoury (Guyane), p. 107-128.

Charbit Y. et Chort I., 2006, Les transferts monétaires des migrants : pays industrialisés etpays en développement. Revue européenne des migrations internationales, vol. 22, n 2, p. 127-154.

Denour L. et Junker R., 1995, Les médecins étrangers dans les hôpitaux français. Revue européenne des migrations internationales, vol. 11, $n^{\circ} 3, p .145-166$ 
Diouf-Kamara S., 1991, Aperçus américains : les Africains, des « battants » et des intellectuels. Hommes et migrations, $\mathrm{n}^{\circ} 1149$, p. 20-26.

Girondin K., 1986, Les tentatives de peuplement de la Guyane de 1848 à 1914. Equinoxe, nº 21, p. 1-37.

Granger S., 2006, Guyane et Surinam, à l'intersection des migrations caraïbes et sud-américaines. Dynamiques migratoires de la Caraibe, Terrres d'Amérique n 6, GEODE-Caraïbe/Karthala, p.287-302.

Moomou J., Les Bushinenge en Guyane : entre rejet et intégration de la fin du XVIIIè siècle aux dernières décennies du XXè siècle. Comprendre la Guyane d'aujourd'hui, Ibis Rouge Editions, Matoury (Guyane), p. 51-82.

\section{NOTES}

1. L'immigration de travailleurs contractuels de l'Inde, commencée dès 1856 , s'est poursuivi jusqu'en 1877 et a totalisé 8472 personnes.

2. Homme d'affaires très populaire en Guyane, Jean Galmot avait été élu triomphalement lors des élections législatives de 1919. Cependant, aux élections de 1924, son adversaire, soutenu par l'administration coloniale, fut élu député grâce à la fraude électorale. En 1928, l'administration imposa à nouveau son adversaire par les mêmes procédés. Mais la morte suspecte de Galmot en août 1928 déclencha à Cayenne une émeute sanglante.

3. Mam-Lam-Fouck S., Histoire de la Guyane contemporaine (1940-1982), Editions caribéennes, Paris, 447 p. : 67-69.

4. Le plus ancien Africain présent en Guyane est un Guinéen arrivé en 1981, en provenance de Guadeloupe. Un enseignant, marié à une Guyanaise, s'était installé au début des années 1970 mais il est possible qu'il y ait eu des arrivées précédentes.

5. Lors de l'instruction des dossiers, les demandeurs d'asile étaient convoqués à l'OFPRA, à Paris et ils en profitaient pour rester en France mais depuis qu'une antenne a été installée en Guadeloupe, les personnes concernées ne se déplacent plus.

6. Pour survivre, les Africains sans papiers effectuent des «jobs», en particulier au marché de Cayenne où ils s'activent avec leur brouette pour décharger les marchandises des agriculteurs et des revendeuses ou pour transporter les achats des clients. Si cette activité était auparavant relativement rémunératrice, depuis les années 2000, la concurrence est plus vive avec l'augmentation des migrants dans ce secteur, d'autant que des Péruviens, nouveaux venus également en Guyane, se sont installés dans ce créneau. Les tensions ont débouché, à la fin de 2006, sur des bagarres entre Africains et Péruviens, ce qui a entraîné une intervention de la municipalité de Cayenne.

7. Source : enquêtes personnelles.

8. Ibidem.

9. Calmont A., Les originaires d'Afrique subsaharienne aux Antilles françaises, entre migration familiale et exode de cerveaux, article à paraître.

10. Le terme «franco-africain » désigne ici un couple formé d'un conjoint d'origine française métropolitaine et d'un conjoint d'origine africaine.

11. Source : enquêtes personnelles.

12. Source : enquêtes personnelles.

13. Ibidem.

14. Le marabout est à l'origine celui qui possède une connaissance approfondie du Coran et qui, grâce aux vertus du Livre Sacré, est censé avoir une influence sur le cours de la vie et il peut donc être sollicité pour tout. 
15. En Guyane, selon une hiérarchisation largement bâtie sur des stéréotypes, la population est répartie entre les "violents» (Brésiliens, Surinamais, Guyaniens) et les «calmes» (Haïtiens, Hmongs, Chinois), la violence étant perçue comme marqueur de l'identité culturelle d'un groupe ethnique ou national.

16. Les Bushinengue sont les descendants des esclaves marrons du Surinam qui avaient trouvé refuge dans les forêts de l'intérieur, sur les bords du Maroni. Ils sont quelques fois appelés "Africains de Guyane ».

17. Créée en 1939 pour venir en aide aux personnes déplacées par la guerre, la Cimade agit depuis pour l'accueil et l'accompagnement social et juridique des étrangers en France. Elle est notamment la seule association présente dans les centres de rétention où sont enfermés les étrangers contraints de quitter le territoire.

\section{RÉSUMÉS}

Installés en Guyane depuis une vingtaine d'années et encore peu nombreux, les Africains constituent une migration originale, très différente des flux régionaux traditionnels. À côté de l'arrivée de sans papiers peu ou pas qualifiés, existe un flux majoritaire de personnes diplômées, arrivées légalement à partir de la France et culturellement proches de la société d'accueil. À cela s'ajoutent une proximité phénotypique et une faible mobilisation des ressources identitaires pour en faire une immigration invisible qui s'insère sans problème majeur dans la société guyanaise.

Settled in French Guyana for 20 years and still not very many, Africans represent an original migration, very different from traditional regional flows. Beside the arrival of undocumented people little or no qualified, there is a majority flow of graduates, legally proceeding from France and culturally close to the host society. In addition, we can add a phenotypic proximity and a weak mobilization of identity resources which make of it an imperceptible immigration, fitting without major problems into the French Guyanese community.

\section{INDEX}

Mots-clés : Guyane, Africains, flux migratoires, qualifiés, sans-papiers

Keywords : French Guyana, Africans, migration flows, qualified, undocumented

\section{AUTEUR}

\section{ANDRÉ CALMONT}

André Calmont (andre.calmont@orange.fr) est enseignant chercheur à Université des Antilles et de la Guyane. Il a récemment publié :

- Trajets socio-identitaires chez les jeunes issus de la migration haïtienne en Guyane, 2007, in Dynamiques migratoires de la Caraïbe, Terres d'Amérique, n 6, p 199-220, Paris, Géode CaraïbeKarthala ; 
- Dynamiques migratoires en Guyane : des politiques migratoires de développement au développement des migrations spontanées, 2007, in Comprendre la Guyane d'aujourd'hui, Matoury, Ibis rouge Éditions, p. 107-128. 